\title{
Femtosecond Electronic Thermal Oscillation in Electron Temperature Dynamics in Thin Gold Film
}

\author{
F. M. Jasim ${ }^{1}$,M. I. Azawe \\ Dept. of Physics, Mosul University, Mosul, Iraq
}

\begin{abstract}
High intense femtosecond laser pulse is irradiated on thin gold film. Electron temperature was evaluated using the two-temperature and the dual-phase lag models. Numerical results showed a thermal oscillation in electron temperature dynamics. To assess thermal oscillation, analytical solution of the characteristic equation for heat conduction was obtained by the Laplace transformation method and implied with the Van Neumann condition. More than one temperature locations in the surface of thin gold film were observed.

Another feature was also observed from the numerical solutions was the shallow concentration of temperature distribution of the film. Instabilities and non-homogeneous temperature distribution on front surface of the gold target were also studied in terms of plume expansion. Plume expansion (in vacuum) in terms of Knudsen layer thickness will be presented for different laser fluence and pulse duration. These results suggest that the laser parameters can influence the plume characteristics. Plume expansion in two dimensions of the surface exposed to the laser will be presented in order to show that the plume expansion was abroad and can be interpreted in terms of laser fluence.
\end{abstract}

Keywords - femtosecond laser pulse, gas dynamics, laser plume expansion, thin film gold foil, two-temperature model.

\section{Introduction}

Laser pulse of very short duration irradiation to a very small geometrical dimension of gold foil, can produce an extreme temperature gradients in the object. Such an ultrafast heating of the thin gold film can be well described by the so-called two- temperature model, initial conditions and boundary-valued partial differential equations [1]. Theory of heat transmission based on Fourier's law predicts infinite propagation speed heat disturbances. This law relates heat flux and the temperature gradient. According to this law, heat flux adjusts immediately to the imposed temperature gradient, i.e., there is no relaxation time for the heat flux [2]. So, one has to consider the lag between the heat flux and the temperature gradient. This lagging behavior in the transient process is caused by the finite time required for the microscopic interactions to take place [3]. In 1995, Tzou [4] proposed the dual-phase lag (DPL) model to explain the non-Fourier heat conduction phenomenon found in many mediums. In this model, i.e., the dual lags, the heat flux time lag and the temperature gradient time lag, supersedes the Fourier law for micro-scale heat transport mechanisms. Micro-scale heat transport is governed by electron-lattice interaction in metallic films. The parabolic two-step model [5] can express energy transport equations for the flow of heat from the hot electrons to the lattice. The model takes into account the laser energy absorption by the conduction band electrons, energy transfer from hot electrons to the atomic vibrations due to the electron-phonon coupling, and the electronic heat diffusion from the irradiated surface to the bulk of the target [6]. Quintanilla and Racke studied the theory of the heat conduction model with threephase-lag [7].

Nonequilibrium thermal distribution had been subjected to intensive research efforts due to the above applications and to establish a theoretical explanation of thermal transport of both subsystems (electrons and phonons). Many research works have demonstrated that Fourier's Law of heat conduction is not suited for modeling ultrafast heat transport processes, in which the energy excitation times are shorter than or comparable to the relaxation times of heat carriers [8]. For this reason, the two temperature models (TTM) have been employed for describing ultrashort laser-metal interactions [9], and the TTM was revised by Qiu and Tien [10] based on Boltzmann transport equation. They showed that in the short time-step heating regime, the microscopic energy transfer among photon, electrons, and phonons enlarges the size of the heat affected region and lowers the lattice temperature rise significantly.

In this article, the two-temperature model (TTM) will be considered for conduction heat transfer, where the ballistic nature of electrons and phonons is maintained in the governing equations. When the thickness of the target is less than the electron mean free path, ballistic electron transport would lead to electron-phonon 
coupling. The general TTM can be simplified to produce the so-called hyperbolic TTM, parabolic TTM, and even the dual-phase lag model. Interesting features of the electron energy exchange will be presented and interpreted as transient or instabilities or even oscillating thermalization to the surroundings.

\section{Theory}

Energy transport after a very short laser pulse (in femtoseconds) absorption will be given. The equation for electron temperature can be written as [11-16]:

$C_{e}\left(T_{e}\right) \cdot \frac{\partial T_{e}}{\partial t}=\nabla \cdot\left(K_{e}\left(T_{e}\right) \cdot \nabla T_{e}\right)-G\left[T_{e}-T_{l}\right]+s(r, t)$

and the equation for the lattice temperature is:

$$
C_{l}\left(T_{l}\right) \cdot \frac{\partial T_{l}}{\partial t}=G\left[T_{e}-T_{l}\right]
$$

where $\mathrm{C}, \mathrm{T}$, and $\mathrm{G}$ are the specific heat capacity, temperature, and electron-phonon coupling, respectively. The subscripts e and 1 stand for the electron and lattice, respectively. $s$ is the heat deposition rate due to laser source radiation absorption. $K_{e}$ is the effective electron thermal conductivity which accounts for electronelectron and electron-phonon scatterings, and is defined as [17,18]:

$$
K_{e}\left(T_{e}\right)=K_{0} \cdot\left(\frac{T_{e}}{T_{l}}\right)
$$

The value of $K_{0}$ used in the calculations is $315 \mathrm{~W} \mathrm{~m}^{-1} \mathrm{~K}^{-1}$ [19].

The electronic specific heat capacity is taken to be proportional to the electron temperature as [20,21]:

$$
C_{e}\left(T_{e}\right)=\gamma T_{e}
$$

where $\gamma$ is the coefficient for electronic heat capacity. The value of $\gamma$ for gold used in the calculations is $71 \mathrm{~J}$ $\mathrm{m}^{-3} \mathrm{~K}^{-2}$ [22]. A value of $2.5 \times 10^{6} \mathrm{Jm}^{-3} \mathrm{~K}^{-1}$ for $C_{l}$ will be used in the computations here.

The electron-phonon coupling $\mathrm{G}$ has been under active investigations in the field of superconductivity for several decades and now has attracted renewed interest in femtosecond laser applications [22]. A constant value for the electron-phonon coupling is used as for low electronic temperatures, rather than temperature dependence. The value of $\left(2.2 \times 10^{16} \mathrm{Wm}^{-3} \mathrm{~K}^{-1}\right)$, which was obtained from reflectivity experiments by Ref. [23], will be considered in our calculations for the electron-phonon coupling $\mathrm{G}$. The ratio of $\left(C_{e} / \mathrm{G}\right)$ is called the time constant for the electron-phonon interaction, and for Au it is $0.75 \mathrm{ps}$ [5].

The laser source $s(x, z, t)$ can affect both the electron and lattice temperatures and hence the laser heat source has to be characterized properly, i.e., a flat-top laser beam (uniform intensity over the entire laser spot) is considered [24]. The following assumption has to be made that the excited electrons are immediately and fully thermalized when the laser pulse is applied [25]. The laser source (power density in $\mathrm{W} / \mathrm{m}^{3}$ ) can be written as [26]:

$$
s(x, z, t)=\sqrt{\frac{B}{\pi}} \frac{(1-R) J_{0}}{t_{p} \delta}\left[1+\alpha \cos \left(\frac{2 x}{L} \pi\right)\right] \cdot \exp \left[-\frac{z}{\delta}-B\left(\frac{t-2 t_{p}}{t_{p}}\right)^{2}\right]
$$

where $J_{0}$ is the laser fluence $\left(\mathrm{J} \mathrm{m}^{-2}\right), R$ is the gold surface reflectivity $(R=0.93[25]), t_{p}$ is the laser pulse duration ( $\left.t_{p}=100 \mathrm{fs}[25]\right)$ at FWHM, , $\delta(=15.3 \mathrm{~nm}$ [25]) is the optical penetration depth of photons in gold (where the intensity amplitude is decreased by a factor e), $L$ is the spatial period with $(-L / 2 \leq x \leq L / 2)$ and $z$ is the depth coordinate of gold film. $\alpha$ is the coefficient of the periodic amplitude $(0 \leq \alpha \leq 1)$, and $B=4 \ln (2) \quad$, is the normalization factor that describes the temporal Gaussian pulse function [26]. In other words, the laser source is assumed a cosine function along the surface (along $\mathrm{x}$-axis) and attenuates exponentially into the gold skin depth (along z-axis). Lasing is assumed to start at $\mathrm{t}=0$ and ends at $t=4 t_{p}$, and the laser energy outside this period of time is neglected since it is too small to significantly to affect the electron temperature [25]. The initial values and boundary conditions of the TTM are as follows. The initial conditions for both electrons and phonons are: 
$T_{e}\left(z,-2 t_{p}\right)=T_{l}\left(z,-2 t_{p}\right)=T_{o}$

While the boundary conditions, assuming that the gold film front and back surfaces were thermally insulated:

$\frac{\partial T_{e}}{\partial x}(0, t)=\frac{\partial T_{e}}{\partial x}(L, t)=\frac{\partial T_{l}}{\partial x}(0, t)=\frac{\partial T_{l}}{\partial x}(L, t)=0$

The characteristic equation of thermal transport taking into account the dual phase lag under the influence of laser heat pulse can be written as [27]:

$\frac{\tau_{q}}{\kappa} \frac{\partial^{2} T}{\partial t^{2}}+\frac{1}{\kappa} \frac{\partial T}{\partial t}=\frac{\partial^{2} T}{\partial x^{2}}+\tau_{T} \frac{\partial^{3} T}{\partial x^{2} \partial t}+\frac{1}{k}\left(s+\tau_{q} \frac{\partial s}{\partial t}\right)$

where $\tau_{q}$ is the relaxation time of heat flux, $\tau_{T}$ is the relaxation time of the temperature gradient, $k$ is the electron thermal conductivity.

The laser heat source, $s$, is expressed as [27]:

$s(x, t)=I(t)(1-R) \mu \exp (-\mu x)$

where the laser source is assumed to be effective in the surface of the gold film, since we are interested in the electron temperature distribution rather than skin deep of the film. $\mu$ is the absorption coefficient of gold. The laser heat source $s\left(\mathrm{Wm}^{-3}\right)$ is assumed to be Gaussian and had been separated in variables in time and dimension.

Equation (9) can be written in a dimensionless form as:

$$
s(x, t)=I(t)(1-R) \mu \exp (-\beta X)
$$

where $\beta$ is the dimensionless absorption coefficient, given by:

$$
\beta=2 w t_{k} \mu
$$

$w\left(=\alpha / \tau_{q}\right)$ is the speed of thermal heat wave propagation (sound wave) and $\alpha$ is the thermal diffusivity of gold $\left(=k /\left(\rho C_{p}\right)\right)$.

Equation (8) can be written as:

$$
\tau_{q} \frac{\partial^{2} T}{\partial t^{2}}+\frac{\partial T}{\partial t}=\kappa \frac{\partial^{2} T}{\partial x^{2}}+\kappa \tau_{T} \frac{\partial^{3} T}{\partial x^{2} \partial t}+\frac{1}{\rho c_{p}}\left(s+\tau_{q} \frac{\partial s}{\partial t}\right)
$$

The following variables were adopted and defined as:

$$
\chi=\frac{\omega x}{2 \kappa} \quad, \quad \tau=\frac{t}{2 \tau_{q}} \quad, \quad \psi=\frac{s \tau_{q}}{\rho c_{p}\left(T_{m}-T_{o}\right)} \quad, \quad \theta=\frac{T-T_{o}}{T_{m}-T_{o}}
$$

Equation (12) is expressed in terms of the above dimensionless variables as:

$$
\frac{\partial^{2} \theta}{\partial \tau^{2}}+2 \frac{\partial \theta}{\partial \tau}=\frac{\partial^{2} \theta}{\partial \chi^{2}}+\frac{\tau_{T}}{2 \tau_{q}} \frac{\partial^{3} \theta}{\partial \chi^{2} \partial \tau}+2 \frac{\partial \psi}{\partial \tau}+4 \psi
$$

The function $\psi$ will be given in the following equation when the laser source had been taken in to account:

$$
\psi(\chi, \tau)=\frac{\tau_{q}}{\rho c_{p}\left(T_{m}-T_{o}\right)} I\left(2 \tau \tau_{q}\right)(1-R) \mu \exp (-\beta \chi)
$$

To modify the laser heat source term $I\left(2 \tau \tau_{q}\right)$ in terms of arbitrary reference laser intensity $I_{r}$ as:

$$
I\left(2 \tau \tau_{q}\right)=I_{r} \eta(\tau)
$$

with $\eta(\tau)$ is the dimensionless rate of energy absorbed in the medium. Hence, the function $\psi$ will be in the form of:

$$
\psi(\chi, \tau)=\frac{\tau_{q}}{\rho c_{p}\left(T_{m}-T_{o}\right)} \eta(\tau) I_{r}(1-R) \mu \exp (-\beta \chi)
$$


In other words, the function $\psi$, can be written as:

$$
\psi(\chi, \tau)=\psi_{o} \eta_{(\tau)} \exp (-\beta \chi)
$$

With $\psi_{o}$ is given by:

$$
\psi_{o}=\frac{I_{r}(1-R) \mu \tau_{q}}{\rho c_{p}\left(T_{m}-T_{o}\right)}
$$

The characteristic equation, given by Eq. (13), will be using the above substitutions as:

$$
\frac{\partial^{2} \theta}{\partial \tau^{2}}+2 \frac{\partial \theta}{\partial \tau}=\frac{\partial^{2} \theta}{\partial \chi^{2}}+\frac{\tau_{T}}{2 \tau_{q}} \frac{\partial^{3} \theta}{\partial \chi^{2} \partial \tau}+2 \psi_{o}\left(\frac{\partial \eta}{\partial \tau}+2 \eta\right) \exp (-\beta \chi)
$$

Now, the initial dimensionless conditions will be:

$\theta(\chi, 0)=0$

$\frac{\partial \theta}{\partial \tau}(\chi, 0)=2 \psi=2 \psi_{0} \eta(0) \exp (-\beta \chi)$

The dimensionless boundary conditions for our present problem are:

$\frac{\partial \theta}{\partial \tau}(0, \tau)=0$

$\frac{\partial \theta}{\partial \tau}(L, \tau)=0$

The Laplace transformation of the characteristic equation (Eq.(19)), will be [22]:

$$
\frac{d^{2} \bar{\theta}_{l}(\chi, u)}{d \chi^{2}}-u(u+2) \bar{\theta}_{l}(\chi, u)=-2 \psi_{0}(u+2) \bar{\eta}(u) \exp (-\beta \chi)
$$

This equation had been obtained using the above dimensionless initial conditions given by Eq.(13) and Eq. (14). Knowing that the Laplace transforms are:

$$
\begin{aligned}
& \bar{\theta}_{l}(\chi, u)=L[\theta(\chi, \tau)] \\
& \bar{\eta}(u)=L[\eta(\tau)]
\end{aligned}
$$

The satisfying boundary conditions are given by:

$$
\begin{aligned}
& \frac{d \bar{\theta}_{l}}{d \chi}(0, u)=0 \\
& \frac{d \bar{\theta}_{l}}{d \chi}(L, u)=0
\end{aligned}
$$

We had assumed that the target is isolated.

The analytical solution (Van Neumann condition) of the characteristic equation (Eq.(24)), taking in to account the above boundary conditions is of the form:

$$
\bar{\theta}_{l}(\chi, u)=c_{2} \sin (\lambda \chi)+c_{3} \exp (-\beta \chi)
$$

with $\lambda$ is given by:

$$
\lambda^{2}=-[u(u+2)]
$$

where $u$ is the Laplace variable.

Substituting Eq. (29) in to Eq.(24) we get: 
$-c_{2} \lambda^{2} \sin (\lambda x)+c_{3} \beta^{2} \exp (-\beta x)+\lambda^{2}\left[\begin{array}{l}c_{2} \sin (\lambda x) \\ +c_{3} \exp (-\beta x)\end{array}\right]=-2 \psi_{0} \eta(s+2) \exp (-\beta x)$

Resulting from the above equation is the following equation for the constant $c_{3}$ :

$$
c_{3}=\frac{2 \psi_{0} \bar{\eta}(u+2)}{u(u+2)-\beta^{2}}
$$

In order to calculate the constant $c_{2}$, we had solved the analytical solution for the boundary condition as:

$$
\frac{d \theta}{d \chi}(\chi=l)=0=c_{2} \lambda \cos (\lambda l)+c_{3}(-\beta) \exp (-\beta l)
$$

We get:

$$
c_{2}=\frac{2 \beta \psi_{0} \bar{\eta}(u+2) \exp (-\beta l)}{\left(u(u+2)-\beta^{2}\right)[-u(u+2)]^{\frac{1}{2}} \cos (\lambda l)}
$$

Finally we get the following equation that is to be solved numerically

$$
\bar{\theta}(\chi, u)=\frac{2 \beta \psi_{0} \bar{\eta}(u+2) \exp (-\beta l)}{\left(u(u+2)-\beta^{2}\right)[-u(u+2)]^{\frac{1}{2}} \cos (\lambda l)} \sin (\lambda \chi)+\frac{2 \psi_{0} \bar{\eta}(u+2)}{u(u+2)-\beta^{2}} \exp (-\beta \chi)
$$

The equation of laser plume gas dynamics represents the surface temperature distribution for the plume of gold film irradiated by Gaussian femtosecond laser pulse [28] :

$T(r, t, z)=\frac{F_{\max }}{\alpha}\left(\frac{\kappa}{\pi}\right)^{1 / 2} \int_{0}^{t} \frac{p\left(t-t^{\prime}\right) d^{2}\left(t^{\prime}\right) d t^{\prime}}{t^{\prime 1 / 2}\left(4 \kappa t^{\prime}+d^{2}\left(t^{\prime}\right)\right)} \exp \left[\frac{-z^{2}}{4 \kappa t^{\prime}}-\frac{r^{2}}{4 \kappa t^{\prime}+d^{2}\left(t^{\prime}\right)}\right]$

with $F_{\max }$ is the laser peak flux density. The symbols given in the above equation is for thermal properties of gold. $\alpha$ is the thermal conductivity, $\kappa=\alpha / \rho c$ is the thermal diffusivity, $\rho$ is density, $\mathrm{c}$ is heat capacity . $p(t)$ is the normalized pulse magnitude $(0 \leq p(t) \leq 1)$.

\section{Results And Discussion}

The above equations will be solved numerically with the aid of Matlab for different conditions. We had used typical parameters of the noble metal (gold) as given in Table I.

Table I: Typical parameters of gold used in the simulation

\begin{tabular}{|l|c|l|l|l|l|l|}
\hline Property & $\propto$ & $\mu$ & $\mathrm{K}$ & $\mathrm{L}$ & $T_{\circ}$ & $w$ \\
\hline Definition & $\begin{array}{l}\text { Thermal } \\
\text { diffusivity }\end{array}$ & $\begin{array}{l}\text { Absorption } \\
\text { coefficient }\end{array}$ & $\begin{array}{l}\text { Thermal } \\
\text { conductivity }\end{array}$ & $\begin{array}{l}\text { Film } \\
\text { thickness }\end{array}$ & $\begin{array}{l}\text { Ambient } \\
\text { temperature }\end{array}$ & $\begin{array}{l}\text { Thermal } \\
\text { wave speed }\end{array}$ \\
\hline $\begin{array}{l}\text { Numerical } \\
\text { value }\end{array}$ & $\begin{array}{l}1.2 \times 10^{-4} \\
\mathrm{~m}^{2} / \mathrm{s}\end{array}$ & $1 \times 10^{9} \mathrm{~m}^{-1}$ & $315 \mathrm{~W} /(\mathrm{mK})$ & $20 \mathrm{~nm}$ & $300 \mathrm{~K}$ & $1.9085 \mathrm{~m} / \mathrm{s}$ \\
\hline
\end{tabular}

Fig. 1 show the 3D plot after adjusting the grid size from convergence test and time step was set to 0.25 ps to reach numerical analysis consistency. The electron temperature was plotted as a function of time and distance (penetration from the front surface of the gold film). We can notice that the electron temperature reached its maximum value in less than one femtosecond and the complete electron thermalization was $4 \mathrm{~nm}$ in depth of the gold film. This will be studied more in depth and thoroughly when considering the dual phase lag. 


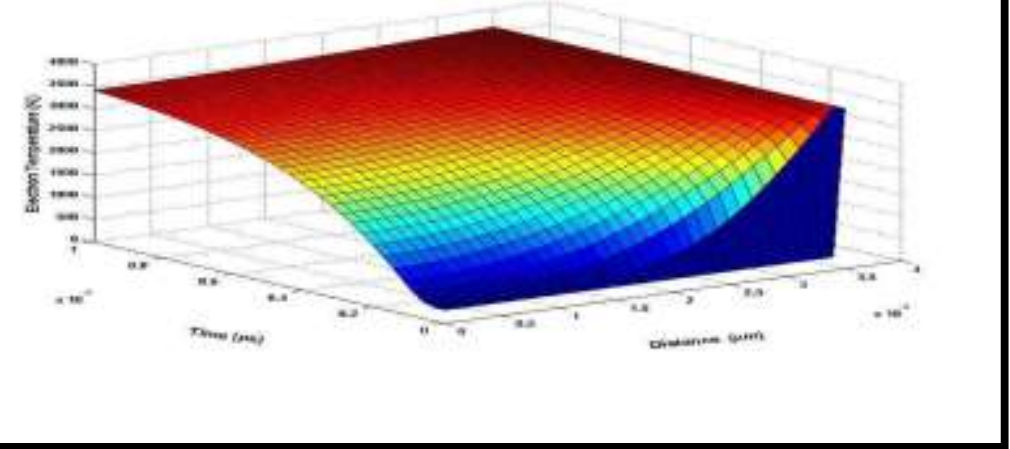

Fig.1 Electron temperature simulation as a function of time and gold skin depth computed for gold film of $20 \mathrm{~nm}$ thickness when irradiated by $13.7 \mathrm{Jm}^{-2}$ laser fluence and $100 \mathrm{fs}$ pulse duration.

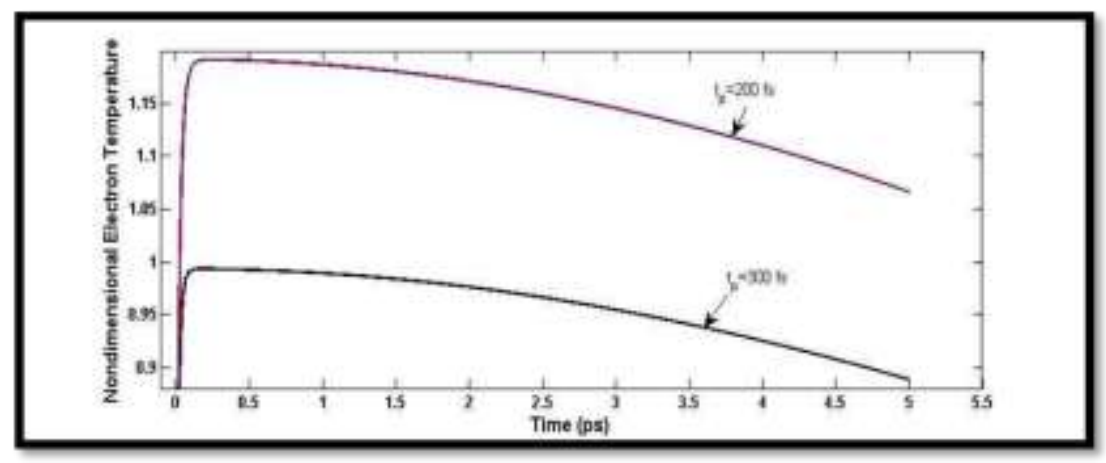

Fig. 2 Numerical simulation of the electron temperature dynamics of gold nanostructure induced by the influence of the variation of laser pulse duration. The simulation was carried out first with $300 \mathrm{fs}$ and then $200 \mathrm{fs}$, laser pulse duration.

To study the influence of pulse duration of the laser on the electron temperature taking into account relaxation times $\tau_{q}$ and $\tau_{t}$ in the simulation, Fig. 2 shows the results of this simulation. Two values of laser pulse duration were taken as (200fs) and (300fs).

The influence of relaxation time on the electron temperature dynamics, Fig. 3, shows this effect. The values of, $\tau_{q}$ (relaxation time of heat flux), and $\tau_{T}$ (the relaxation time of temperature gradient) were taken as shown in the figure. When the simulation was carried out with $\left(\tau_{q} \succ \tau_{T}\right)$, we found an interesting feature for the electron dynamics, that is, the electron temperature started to decrease with oscillating behavior. The oscillations get larger as electron kinetics with time proceeds to lattice exchange. This behavior of electron dynamics, to our knowledge, had not been published. The explanation of this behavior can be interpreted as, $\tau_{q}$ means a finite time is required to raise the temperature of electrons, while $\tau_{T}$ indicates the delay that exists in electron-phonon exchange. This delay is shown as oscillations.

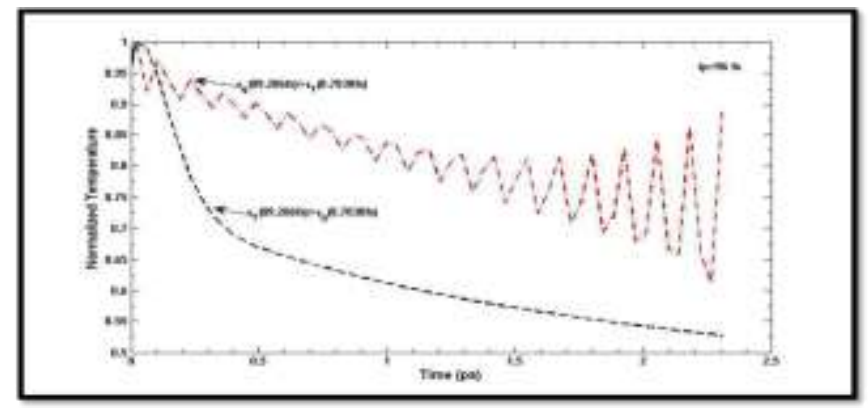

Fig. 3 The time evolution of electron temperature for two cases, showing oscillations when $\tau_{q} \succ \tau_{T}$. Laser irradiation of front surface of the nanostructure gold film was with fluence $13.7 \mathrm{Jm}^{-2}$ and pulse duration of $96 \mathrm{fs}$. 
The temperature distribution on the front surface of nanostructure gold film when irradiated by a femtosecond laser pulse will be studied numerically with the help of (DPL) model. The simulation was performed using FDTD (finite-difference time-domain) algorithm to solve the characteristic equation of heat conduction. The time increment for simulation and grid size were adjusted until the stability and convergence criteria were obtained satisfactory, giving time step few femtoseconds and spatial increment of $3 \mathrm{~nm}$. Many mesh grid size were tested in simulation, until the above condition was reached.

Fig. 4 shows the numerical simulation of temperature distribution of the front surface of the gold film irradiated by ( $96 \mathrm{fs})$ laser pulse duration and fluence of $\left(13.7 \mathrm{Jm}^{-2}\right)$ by solving the characteristic equation of DPL model.

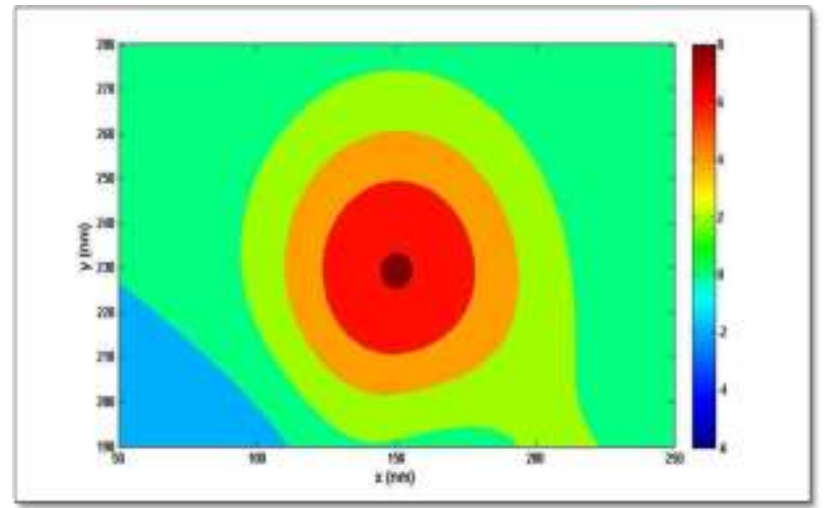

Fig. 4 Temperature distribution of the front surface of gold film when exposed to laser Gaussian pulse of duration of (96 fs) and power density of $\mathrm{s}_{0}=5.88 \times 10^{20} \mathrm{Wm}^{-3}$.

The calculations of the dimensionless surface temperature $(\bar{\theta})$ as a function of dimensionless displacement $(\chi)$, for various values of the dimensionless absorption coefficient, while keeping the other variables $\eta$ and $\psi_{o}$ as constants is shown in Fig. 5. It is obvious from the figure that for small values of $\beta$ the temperature distribution extends more than for greater value of $\beta$. This behavior needs no explanation as can be predicted from the simple law of absorption for certain material.

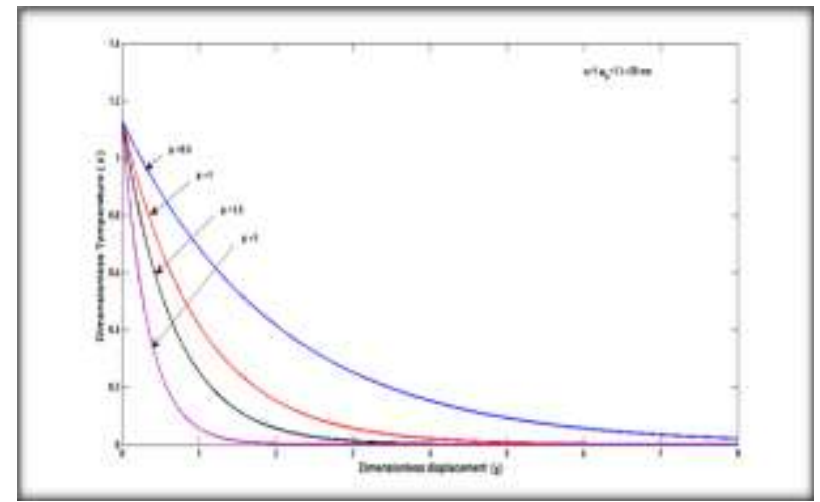

Fig.5 Dimensionless surface temperature as a function of dimensionless displacement for different values of dimensionless absorption coefficient $(0.5,1,1.5,3)$ and keeping dimensionless rate of energy absorbed in the medium constant $(\eta)$ and dimensionless internal heat source also constant $\left(\psi_{o}\right)$. 


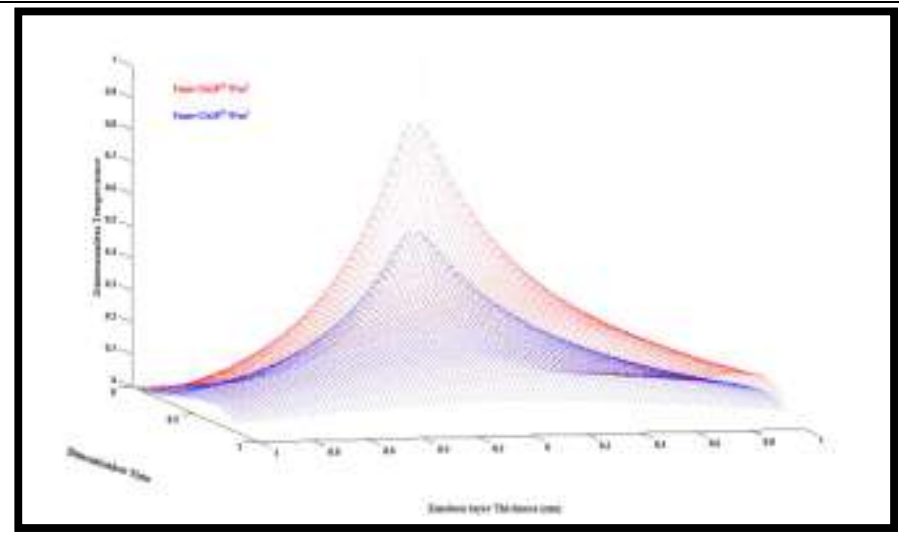

Fig. 6 Illustrates the expansion of plume (in terms of temperature distribution of vapour plume) for two different values of laser fluence of $13.4 \& 23.4 \mathrm{~J} / \mathrm{m}^{2}$ as a function of kundsen layer thickness.

The expansion of the plume was taken into account the effects of laser fluence in order to show how this can influence the expansion in terms of Knudsen layer thickness which had been evaporated by thermal effect of laser pulse, Fig. 6. Laser ablation has to conceder this result in its application.

Laser spot radius was found to be effective on the plume expansion as shown in Fig. 7. Two values were used in the numerical simulation. As can be seen from the graph, higher value of laser spot gives higher temperature distribution and expansion.

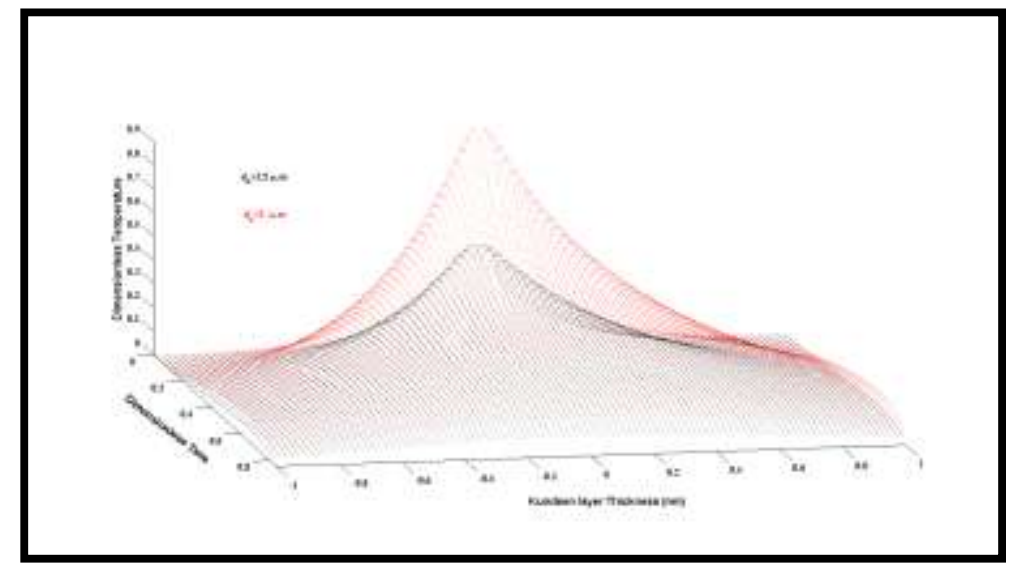

Fig. 7 Representce the influence of laser spot diameter on the temperature distribution of the plume in terms of Knudsen layer thickness.

\section{Conclusions}

Numerical simulation for the heated gold film had been carried out using TTM and DPL to obtain the electron temperature. TTM numerical results showed that electron temperature had slow time decrease in comparison to DPL. Simulations of the characteristic equation (DPL) by FDTD algorithm to study the electron temperature $\left(T_{e}\right)$ taking into account the relaxation times reveals that $T_{e}$ can be affected by laser fluence as well as laser pulse duration. A thermal oscillation in $\mathrm{T}_{\mathrm{e}}$ dynamics was found when $\tau_{q} \succ \tau_{T}$. A temperature distribution on the front surface of the gold film was studied showing part of the surface was extremely at high temperature besides zones of lower temperatures.

The problem of the characteristic equation of thermal transport, taking into account the DPL for thin gold film and irradiated by femtosecond laser pulse, was solved analytically by the Laplace transform method for the case of IVBP.

Dimensionless surface temperature with respect to the dimensionless displacement for various values of the dependent parameters which are; the dimensionless internal heat source, the dimensionless rate of energy absorbed in the medium, and the dimensionless absorption coefficient were studied.

Laser plume expansion from thin gold film was investigated in this article in terms of Knudsen layer for Adiabatic, non-isothermal case of gas dynamics. The results showed that the plume expansion was 
completely dependent on the laser pulse parameters, the pulse duration, fluence, and beam waist. This dependence had been shown implicitly in the plots given. The analytical equations were solved numerically for special case as spatially constant temperature of the laser plume.

\section{References:}

[1] E. Majchrzak and J. Poteralska, Archives of Foundry Engineering , 10(4), 123(2010).

[2] V. V. Kulish and V. B. Novozhilov, Journal of Heat Transfer, 126,805(2004).

[3] J. Ordonez-Miranda and J. J. Alvarado-Gil, Nanoscale Research Letters, 6, 327(2011).

[4] D. Y. Tzou, ASME J. Heat Transf. , 117, 8(1995).

[5] D. Y. Tzou, Macro to Micro-scale Heat Transfer :The Lagging Behavior, chap. 2, Taylor \& Francis, Washington, DC(1996).

[6] Z. Lin and L. V. Zhigilei, Appl. Surface Scien. , 253, 6295(2007).

[7] R. Quintanilla and R. Racke, Int. J. Heat and Mass Transf, 51, 24(2008).

[8] G. X. Wang, V. Prasad "Microscale Heat and Mass Transfer and Nonequilibrium Phase Change in Rapid Solidification," Mater. Sci. Eng. A, 292(2), 142-148 (2000).

[9] S. I. Anisimov, B. L. Kapeliovich and T. L. Perel'man ," Electron Emission from Metal Surface Exposed to Ultrashort Laser Pulses," Sov. Phys. JETP, 39, 375-377 (1974).

[10] T. Q. Qiu and C. L. Tien ,"Heat Transfer Mechanisms During Short-Pulse Laser Heating of Metals," ASME J. Heat Transfer, 115, 835-837 (1993).

[11] P. E. Hopkins and P. M. Nomis, J. Heat Transfer, 131, 043208(2009).

[12] N. Del Fatti, A. Arbouet, and F. Vallee, Appl. Phys. , B84, 175(2006).

[13] O. L. Muskens, N. Del Fatti, and F. Vallee, Nano Lett. , 6(3), 552(2006).

[14] V. V. Kruglyak, R. J. Hicken, P. Matoussek, and M. Towrie, Phys. Rev. , B75, 035410(2007).

[15] A. Hu, Y. Zhou, and W. W. Duley, The Open Surface Scien. J. , 3,42(2011).

[16] J. H. Huang, K. Baheti, J. K. Chen, and Y. Zhang, Front, Heat and Mass Transf. (FHMT), 2, 013005(2011).

[17] H-D. Wang, W-G. Ma, X. Zhang, W. Wang, and Z-Y Guo, InternationalJournal of Heat and Mass Transfer, 54,967 (2011).

[18] S.I. Anisimov, B. Rethfeld, Nonresonant Laser Matter Interact., 92 ,3093 (1997).

[19] X. Wang and X. Xu, J. Thermal Stresses, 25,457 (2002).

[20] I.H. Chowdhury and X. Xu, Numerical Heat Transf., part A 44, 219 (2003).

[21] B. Palpant, Y. Guillet, M. R. Huyeh, and D. Prot, 41(2),105 (2008).

[22] Z. Lin and L. Zhigilie, Proc. SPIE, 6261, (2006).

[23] J. Hohlfeld, S.-S. Wellershoff, J. Gudde, U. Conrad, V. Jahnke, and E.Matthias, Chem. Phys., 251,237 (2000).

[24] J.K. Chen, J.E. Beraun, L.E. Grimes, and D.Y. Tzou, Int. J. Solids and Structures, 39,3199 (2002)

[25] J.K. Chen, D.Y. Tzou and J.E. Beraun, Int. J. Heat and MassTransfer, 49,307 (2006).

[26] J. Wang and C. Guo, J. Appl. Phys. , 102, 053522 (2007).

[27] R. T. AL-Khairy and Z.M. AL-Ofey "'Analytical Solution of the Hyperbolic Heat Conduction Equation for Moving Semi-Infinite Medium under the Effect of Time-Dependent Laser Heat Source,” J. appl. Math., Article ID 604695, (2009).

[28] J. Chen , L.He , D. F. Farson, and S. I .Rokhlin ," experiments and simulations for small-scale electrical discharges", Welding Journal 90,161 (2011) 